\title{
24-Month Phase I/II Clinical Trial of Bimatoprost Sustained-Release Implant (Bimatoprost SR) in Glaucoma Patients
}

\author{
E. Randy Craven ${ }^{1}$ (1) - Thomas Walters ${ }^{2}$ - William C. Christie ${ }^{3} \cdot$ Douglas G. Day $^{4} \cdot$ Richard A. Lewis $^{5}$. \\ Margot L. Goodkin ${ }^{6} \cdot$ Michelle Chen $^{6} \cdot$ Veronica Wangsadipura ${ }^{6} \cdot$ Michael R. Robinson $^{6} \cdot$ Marina Bejanian $^{6} \cdot$ for the \\ Bimatoprost SR Study Group
}

Published online: 28 December 2019

(c) The Author(s) 2019

\begin{abstract}
Objective The objective of this study was to evaluate the safety and intraocular pressure (IOP)-lowering effects over 24 months of biodegradable bimatoprost sustained-release implant (Bimatoprost SR) administration versus topical bimatoprost $0.03 \%$ in patients with open-angle glaucoma (OAG).

Methods This was a phase I/II, prospective, 24-month, dose-ranging, paired-eye controlled clinical trial. At baseline following washout, adult patients with OAG $(N=75)$ received Bimatoprost SR $(6,10,15$, or $20 \mu \mathrm{g})$ intracamerally in the study eye; the fellow eye received topical bimatoprost $0.03 \%$ once daily. Rescue topical IOP-lowering medication or single repeat administration with implant was permitted. The primary endpoint was IOP change from baseline. Safety measures included adverse events (AEs).

Results At month 24, mean IOP reduction from baseline was 7.5, 7.3, 7.3, and $8.9 \mathrm{mmHg}$ in eyes treated with Bimatoprost SR $6,10,15$, and $20 \mu \mathrm{g}$, respectively, versus $8.2 \mathrm{mmHg}$ in pooled fellow eyes; 68,40 , and $28 \%$ of pooled study eyes had not been rescued/retreated at months 6,12 , and 24 , respectively. AEs in study eyes that occurred $\leq 2$ days post-procedure typically were transient. After 2 days post-procedure, overall AE incidence was similar between study and fellow eyes, with some events typically associated with topical prostaglandin analogs having lower incidence in study eyes.

Conclusions Bimatoprost SR showed favorable efficacy and safety profiles up to 24 months, with all evaluated dose strengths demonstrating overall IOP-reducing effects comparable to those of topical bimatoprost. Targeted and sustained delivery of bimatoprost resulted in protracted IOP lowering, suggesting that Bimatoprost SR may represent a transformational new approach to glaucoma therapy. Clinicaltrials.gov identifier: NCT01157364
\end{abstract}

Electronic supplementary material The online version of this article (https://doi.org/10.1007/s40265-019-01248-0) contains supplementary material, which is available to authorized users.

E. Randy Craven

erandycraven@gmail.com

1 Johns Hopkins University School of Medicine, 600 N. Wolfe Street, 110, Baltimore, MD 21287, USA

2 Keystone Research, Ltd, Austin, TX, USA

3 Scott \& Christie and Associates, Pittsburgh, PA, USA

4 Coastal Research Associates, Roswell, GA, USA

5 Sacramento Eye Consultants, Sacramento, CA, USA

6 Allergan plc, Irvine, CA, USA

\section{Key Points}

A single administration of bimatoprost sustained-release implant (Bimatoprost SR) lowered intraocular pressure for up to 1 year in $40 \%$ of patients and up to 2 years in $28 \%$, with no additional treatment.

Efficacy of re-administration with a second implant of Bimatoprost SR was similar to that with the first implant.

The safety profile of Bimatoprost SR was favorable during the 24-month study. 


\section{Introduction}

Glaucoma is a group of progressive diseases characterized by damage to the optic nerve that may lead to vision loss and eventual blindness if left untreated [1, 2]. It is the leading cause of irreversible blindness worldwide, and estimates suggest that glaucoma will affect nearly 80 million people globally by 2020 [2-4]. Glaucoma poses a substantial patient burden that increases with disease progression [5-7]. Individuals may experience decreased quality of life because of reduced mobility and ability to perform routine tasks as well as an increased risk of falls and associated injury and death [8-10]. The most common form of glaucoma is open-angle glaucoma (OAG), accounting for $74 \%$ of people diagnosed globally [3].

To date, elevated intraocular pressure (IOP) is the only known modifiable risk factor that, when treated, can delay progression of OAG [2]. Because of the chronic, slowly progressing nature of $\mathrm{OAG}$, preservation of vision requires maintenance of an effective IOP-lowering regimen throughout a patient's lifetime $[1,2]$. Typically, first-line treatment for OAG consists of topical eye drops containing IOP-lowering agents such as prostaglandin analogs/prostamides (PGAs) or $\beta$-blockers. When drops fail to control IOP adequately or are not preferred, laser trabeculoplasty (e.g., selective laser trabeculoplasty) and/or surgery (e.g., minimally invasive glaucoma surgery, invasive procedures such as trabeculectomy, traditional tube shunt) are considered for advancement of care $[1,2]$.

Bimatoprost is a member of the PGA class of IOPlowering medications often used as first-line treatment of primary OAG. IOP is modulated in the anterior segment of the eye by balancing the production of aqueous humor by the ciliary body epithelium and its drainage through two outflow pathways: the conventional trabecular and unconventional uveoscleral pathways. Of the two pathways, the conventional trabecular meshwork pathway is specifically associated with increased flow resistance in glaucoma [11].

PGAs reduce IOP by enhancing aqueous humor outflow [12]. While it is better understood how PGAs modify outflow through the uveoscleral pathway, evidence suggests that PGAs may also act on the trabecular pathway by promoting changes in the extracellular matrix, leading to tissue remodeling through the regulation of matrix metalloproteinases (MMPs; Online Resource 1) [13-17]. The effects of PGAs on MMP expression have also been shown to be concentration dependent [18]. Daily administration of bimatoprost $0.01 \%$ or $0.03 \%$ topical ophthalmic solution has been shown to effectively reduce IOP [19], but poor adherence is common with instillation of topical drops in the treatment of glaucoma [1, 20-23]. Many factors contribute to poor adherence, including forgetfulness, difficulty with instilling eye drops, need for frequent administration, lack of understanding of the disease, the cost of medications, and adverse events (AEs) [23-26]. To reduce the patient treatment burden, bimatoprost was reformulated into a sustained-release (Bimatoprost SR) implant to provide a drop-free, alternative drug-delivery option.

Bimatoprost SR is a biodegradable implant for lowering IOP in patients with OAG or ocular hypertension and uses the NOVADUR ${ }^{\circledR}$ (Allergan plc, Dublin, Ireland) drugdelivery system for intracameral drug delivery [27]. The implant was developed to provide non-pulsatile, sustained release of bimatoprost [28]. The implant was designed with various amounts of polymer to influence the time of drug release and biodegradation rates. In vitro drug release from Bimatoprost SR occurs over a 90-day period. In vivo pharmacokinetic data from the $15-\mu \mathrm{g}$ implant in dogs determined that $80.5 \%$ of the bimatoprost load was released by day 51 and $99.8 \%$ by day 80 [30], with nondetectable drug levels by 4.5 months (Allergan, data on file). Implant administration is performed using a prefilled, single-use, 28-gauge applicator system (Fig. 1), as shown in the video animation (Online Resource 2).

After in vitro studies and in vivo dosing studies were completed, a phase I/II human trial was started using various doses and sizes of implants to determine the safety, efficacy, and duration of effect of the various implants. Interim 6-month results of the phase I/II APOLLO trial showed that Bimatoprost SR provided rapid and sustained IOP lowering at all dose strengths $(6,10,15$, and $20 \mu g)$ [28]. At 16 weeks,
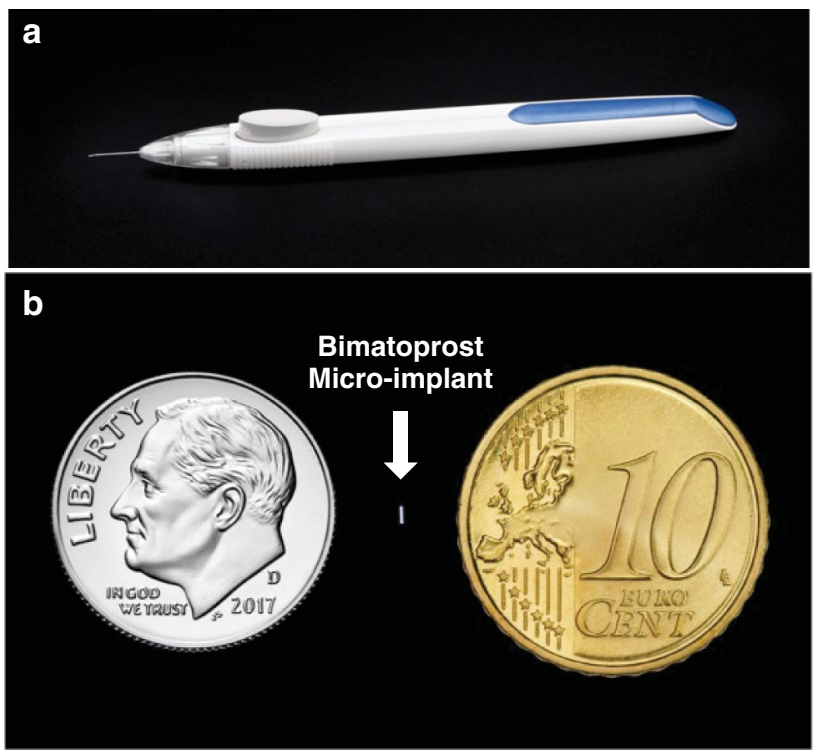

Fig. 1 Bimatoprost sustained-release (SR) single-use implant applicator (a) and photograph of implant next to a dime and Euro for size comparison (b) 
the mean overall IOP reduction ranged, in a dose-dependent manner, from 7.2 to $9.5 \mathrm{mmHg}$; this result was comparable to that observed in topical bimatoprost-treated fellow eyes, which achieved an average $8.4 \mathrm{mmHg}$ overall reduction in IOP. IOP was controlled in the majority of patients with a single administration of Bimatoprost SR for up to 6 months without need for additional treatment. AEs in the study eye were largely reported within the first 2 days after administration; AEs occurring within 2 days were likely related to the administration procedure and were typically transient, resolving quickly. Through 6 months, no serious ocular AEs were reported in study eyes, and no implants had to be removed for safety reasons. Here, we evaluate the long-term, 2-year safety and efficacy results of the completed APOLLO trial of Bimatoprost SR in patients with OAG.

\section{Methods}

\subsection{Study Design}

In this 24-month, phase I/II, open-label, multicenter study, a paired-eye comparison of Bimatoprost SR (four dose strengths) was performed in successive cohorts of patients with OAG (ClinicalTrials.gov identifier NCT01157364). The study followed an adaptive design in which expansion of the ongoing trial was guided by careful consideration of the safety and efficacy data emerging from the trial by a data review committee, which determined the dose strengths to be evaluated as the study progressed [28]. Details of the study design and methodology have been reported previously [28].

Patients were enrolled from 23 September 2010 to 5 August 2014 at 24 clinical sites in six countries: Australia, Canada, Israel, Philippines, Singapore, and the USA. The study was performed in compliance with good clinical practice, the principles of the Declaration of Helsinki, or the laws and regulations of the country in which the study was conducted. Institutional review board or independent ethics committee approval was obtained at each site before the study began, and all patients provided written informed consent before undergoing any study-related procedure.

\subsection{Study Population}

Detailed patient eligibility criteria were previously described in Lewis et al. [28]. Briefly, patients aged $\geq 18$ years were eligible to participate if they had a diagnosis of OAG with $>1 \mathrm{~dB}$ and $<17 \mathrm{~dB}$ of mean deviation visual field loss in the study eye, a history of a minimum of $20 \%$ IOP-lowering response to a topical PGA ocular hypotensive medication, diagnosis of OAG or ocular hypertension in the fellow eye that could be treated adequately with topical bimatoprost
$0.03 \%$ monotherapy, and an iridocorneal angle inferiorly in the study eye of Shaffer grade $\geq 3$ (by gonioscopy) to accommodate the implant without corneal endothelial contact. IOP in both eyes after washout was required to be between 22 and $36 \mathrm{mmHg}$, inclusively, at hour $0(8: 00 \mathrm{a} . \mathrm{m} . \pm 1 \mathrm{~h})$ at the baseline visit. Key inclusion and exclusion criteria are summarized in Online Resource 3. Key exclusion criteria included a history of posterior capsule tear during cataract surgery, any ocular surface finding of greater than trace severity on biomicroscopic examination at baseline, history of conjunctival hyperemia of greater than mild severity with bimatoprost or other PGA use, history of narrow-angle or closed-angle glaucoma, central corneal thickness $<470 \mu \mathrm{m}$ or $>630 \mu \mathrm{m}$ (or a difference between eyes $>70 \mu \mathrm{m}$ ), and central endothelial cell count $<2000$ cells $/ \mathrm{mm}^{2}$ by specular microscopy.

\subsection{Study Treatment}

Patients who were using topical IOP-lowering medication in either eye at screening (days -42 to -4 ) discontinued the medication during the screening period washout window. The required period of washout before the baseline visit was dependent on and prespecified by drug class/product: parasympathomimetic and carbonic anhydrase inhibitor agents, sympathomimetic and $\alpha$-adrenergic agonists, $\beta$-adrenergic antagonists, PGA, and fixed-combination products. If both eyes met the study eye entry criteria at baseline, the eye with the higher IOP (or if the IOP was the same in both eyes, the eye with the larger iridocorneal angle width on optical coherence tomography (OCT) as determined by an independent reading center) was selected as the study eye. Patients were assigned to receive Bimatoprost SR 6, 10, 15, or $20 \mu \mathrm{g}$ and were masked to the dose strength that they received. The site staff who measured IOP were also masked to the study treatment dose strength assignment. On day 1, the Bimatoprost SR implant was administered intracamerally to the study eye using the preloaded, single-use applicator described above. The 6-, 10-, and 15- $\mu$ g dose strengths were available as a single implant that varied in size; two $10-\mu \mathrm{g}$ implants were administered concurrently via the same applicator to achieve the $20-\mu \mathrm{g}$ dose strength. The study eye was prepared for administration according to standard clinical practice: a broad-spectrum topical antibiotic and a topical anesthetic were administered, and the eye was prepped with $5 \%$ povidone-iodine ophthalmic solution and draped in the usual manner for sterile intraocular procedures. The entrance site for the applicator needle was anterior to the insertion of the conjunctiva through the clear cornea in the superior or temporal quadrant of the cornea, with the trajectory of the needle parallel to the iris plane. After administration, the needle track was checked for aqueous leakage and to ensure 
it was self-sealing, and a topical broad-spectrum antibiotic was applied.

Patients were instructed to instill 1 drop of bimatoprost $0.03 \%$ ophthalmic solution in the fellow (control) eye once daily in the evening throughout the 2-year duration of the study. Rescue treatment using topical IOP-lowering drops could be initiated in any eye (study and/or fellow eyes) at the investigator's discretion if the eye failed to attain the target IOP (as determined by the investigator) on consecutive visits $\geq 1$ week apart or if it was considered to be in the best interest of the patient.

A data review committee assessed the ongoing safety in patients who received one or two (20- $\mu$ g dose strength) implant(s) and concluded it was safe to administer an additional implant if needed for IOP control. As a result, a protocol amendment was implemented in 2013 allowing patients who had received a $\leq 15-\mu \mathrm{g}$ dose strength of Bimatoprost SR to receive a single repeat treatment with the same dose strength in the study eye between day 90 and month 12 (Online Resource 4) if they met all of the following criteria: neither eye had received rescue therapy, the study eye failed to maintain a $\geq 20 \%$ reduction in IOP from baseline at 8:00 a.m. on consecutive visits $\geq 1$ week apart, and the initial implant demonstrated adequate safety and did not contact the corneal endothelium. Patients who received a repeat treatment with implant had follow-up visits for a minimum of 12 months after the second administration. Patients could complete the study and be exited at or after the month 18 visit if they had received rescue treatment in the study eye and the implant(s) were not visible to the investigator on gonioscopic examination.

\subsection{Assessments}

IOP was measured at 8:00 a.m. by Goldmann applanation tonometry at all visits using a two-person masked reading method. At baseline, weeks 4, 8, 12, 16, and 20, and month 6, additional measurements were taken at 10:00 a.m., 12:00 p.m., 2:00 p.m., and 4:00 p.m. Patients who had not been rescued in the study eye had additional diurnal measurements at months $9,12,15,18,21$, and 24.

Safety assessments included AEs, biomicroscopy, ophthalmoscopy, macroscopic conjunctival hyperemia, iris color, gonioscopy, visual acuity, visual fields, central corneal endothelial cell density (CECD) by specular microscopy, corneal thickness by pachymetry, and OCT of the macula.

Patients completed a questionnaire on day 8 and week 4 after the first and second (when applicable) administrations. This questionnaire assessed the burdensomeness of the study eye procedure compared with patient expectations. Possible answers were that the procedure was much less burdensome, somewhat less burdensome, as burdensome, somewhat more burdensome, and much more burdensome than expected. A second questionnaire administered at week 12 (after the first administration) and at month 24 or early exit assessed the likelihood that the patient would agree to receive another implant if given the choice and the likelihood that the patient would recommend the implant to individuals with the same disorder. Possible answers were extremely likely, very likely, somewhat unlikely, very unlikely, and extremely unlikely.

\subsection{Outcome Measures and Statistical Analyses}

The primary efficacy measure was IOP, and the primary endpoint was hour-matched IOP change from baseline through 24 months. In the preplanned primary analysis of IOP, data from an eye were censored once the eye received rescue topical IOP-lowering medication or repeat administration with Bimatoprost SR. Further analyses used all available IOP data. The overall mean reduction from baseline IOP during the first 16 weeks of the study was calculated using all observations weighted equally and analyzed with onesample $t$ tests comparing the overall mean reduction from baseline to 0

Secondary endpoints included the mean IOP, use of IOP-lowering rescue treatment or second Bimatoprost SR administration, and patient-reported outcomes. The number of rescue medications used in the study and fellow eye at each time point was also evaluated.

All analyses were performed using SAS software version 9.3 or newer (SAS Institute Inc., Cary, NC, USA). All analyses of IOP used observed data with no imputation for missing data in the modified intent-to-treat population of all treated patients with at least one IOP measurement at baseline and at least one post-baseline IOP measurement through week 16. Data were censored at rescue/retreatment in some analyses. Safety parameters were evaluated using observed values in the safety population of all patients who received the study treatment.

Sample size determination was conducted by a data review committee, which reviewed the available efficacy and safety data throughout the study and determined the dose strengths of Bimatoprost SR to be evaluated and the number of patients to be enrolled for each dose strength. Because of the adaptive nature of this study, the sample size of 75 patients was determined empirically rather than selected to provide power for statistical comparisons between implant dose strengths or between study and fellow eyes.

\section{Results}

\subsection{Patient Baseline Characteristics and Disposition}

In total, 75 patients were enrolled and received the study treatment, and 63 (84.0\%) completed the study. Reasons for 
study discontinuation included AEs in two (2.7\%) patients (cataract, considered treatment-related; adenocarcinoma, considered unrelated to treatment); personal reasons in two (2.7\%) patients; lack of efficacy in one (1.3\%) patient; loss to follow-up in two (2.7\%) patients; and "other" reasons in five patients (6.7\%). Rates of discontinuation were similar among the 10- and 15- $\mu$ g dose strengths (Online Resource 5).

Demographics and study eye characteristics were similar among the groups treated with different dose strengths (Online Resource 6). All patients were diagnosed with primary OAG in both eyes. Overall, mean \pm standard deviation (SD) age was $63.4 \pm 11.7$ years, $45 \%$ were aged $>65$ years, $51 \%$ were female, and $71 \%$ were White. Iris color was dark (brown or dark brown) in 53\% of study eyes and light in 47\% (Online Resource 6). All patients in the Bimatoprost SR treatment groups required washout of prior medications and had a mean IOP increase of $7.8 \pm 4.0 \mathrm{mmHg}$ from screening to after washout (baseline).

\subsection{Efficacy Outcomes}

All dose strengths of Bimatoprost SR reduced the IOP in study eyes. In all dose groups combined, 44 patients required rescue treatment with eye drops; mean IOP (hour 0 ) prior to initiation of rescue was $23.7 \pm 4.8 \mathrm{mmHg}$ [median $23.3 \mathrm{mmHg}$ (range 14-34)]. Figure 2 shows the primary endpoint of time-matched IOP change from baseline at hour 0 (8:00 a.m.). In the preplanned analysis, data were censored from the start of rescue/retreatment, such that the results reflect IOP lowering of a single Bimatoprost SR administration. A sustained IOP-lowering effect that persisted up to month 24 was seen, with the mean IOP reduction from baseline at 24 months in study eyes that had not been rescued or retreated ranging from 5.7 to $7.4 \mathrm{mmHg}$ across all Bimatoprost SR dose strengths (Fig. 2a).

Subsequent analysis of the IOP change from baseline used all observed IOP data in the modified intent-to-treat population, including patients who required rescue treatment with eye drops (Fig. 2b). Although no statistical comparison of the preplanned (Fig. 2a) and post hoc (Fig. 2b) analyses were performed, the overall results indicated similar IOP reductions from baseline between eyes remaining on the initial treatment and rescued eyes for both the Bimatoprost SR-treated eyes and the pooled fellow eyes treated with topical bimatoprost. Analysis of all available data showed a trend toward numerically larger mean IOP reductions in rescued eyes when compared with results of the censored data analysis (Fig. 2a, b). During the first 16 weeks of the study, a dose response of Bimatoprost SR was generally evident in both analyses (Fig. 2); after week 16, the magnitude of IOP reduction with each dose strength was similar to that of a topical PGA. The 8:00 a.m. mean overall study eye IOP reduction from baseline through week 12 , the period used in the ARTEMIS registration trials (all observations weighted equally), ranged from 7.4 to $9.8 \mathrm{mmHg}$ across the Bimatoprost SR dose strengths, compared with $8.4 \mathrm{mmHg}$ in the pooled fellow eyes treated with topical bimatoprost $0.03 \%$ (Table 1). Mean IOP reductions from baseline at month 24 for all available (observed) data were 7.5, 7.3, 7.3, and $8.9 \mathrm{mmHg}$ for the 6-, 10-, 15-, and 20- $\mu \mathrm{g}$ dose strengths in study eyes, respectively, versus $8.2 \mathrm{mmHg}$ in all pooled fellow eyes, with a mean number of additional medications used (including rescue topical IOP-lowering medications or implant retreatment) of $0.64,0.75,0.67$, and 0.90 in study eyes versus 0.05 in pooled fellow eyes (Fig. 2b).

IOP was controlled without rescue or re-administration in $51(68 \%), 30(40 \%)$, and 21 (28\%) study eyes up to 6, 12, and 24 months, respectively (Fig. 3). Subgroup analysis of the patients who completed the study without receiving rescue or re-administration in the study eye showed persistent IOP-lowering effects of a single implant administration in the absence of additional therapy. Mean IOP in study eyes that were not rescued or retreated was reduced from baseline values of $23.6,23.4,23.2$, and $24.4 \mathrm{mmHg}$ to $18.0,16.0$, 15.9 , and $17.8 \mathrm{mmHg}$ at 24 months after a single administration of Bimatoprost SR $6 \mu \mathrm{g}, 10 \mu \mathrm{g}, 15 \mu \mathrm{g}$, and $20 \mu \mathrm{g}$, respectively (Table 2 ).

Among study eyes that received a second administration of Bimatoprost SR, mean baseline IOP values (hour $0)$ were $25.2(n=6), 25.0(n=8)$, and $26.0(n=10) \mathrm{mmHg}$ for eyes in the $6-\mu \mathrm{g}, 10-\mu \mathrm{g}$, and $15-\mu \mathrm{g}$ dose strength groups, respectively. IOP lowering post-treatment in these eyes was similar after the first and second implants. Mean \pm SD reduction in IOP from baseline (data censored at rescue/retreatment) averaged over 12 weeks after administration was $7.9 \pm 3.6 \mathrm{mmHg}$ after the first implant and $8.4 \pm 4.2 \mathrm{mmHg}$ after the second implant for the combined 6-, 10-, and 15- $\mu \mathrm{g}$ dose strengths.

\subsection{Implant Degradation}

Implants were typically observed to initially swell as they biodegraded, and the majority of those received at the first administration visit (day 1) were estimated by the investigator to be $76-125 \%$ of their initial size at assessments through month 6 . By 12 months, the majority of those implants had either totally biodegraded $(n=6)$ or were estimated to be $\leq 25 \%$ of their initial size $(n=26)$. At month 24 , a total of 34 patients were evaluated with gonioscopy, and the implant administered on day 1 had biodegraded and was no longer visible in $26.5 \%$ (9/34) of these patients. Of all implants administered on day 1 (including two implants per study eye for the 20- $\mu$ g dose strength), 30 implants (in 25 eyes) were still visible at month 24 . Size assessment was missing for $6.7 \%$ (2/30) of these implants; of those assessed, the implant size was estimated to be $\leq 25 \%$ of initial size for 
a

Study Visit

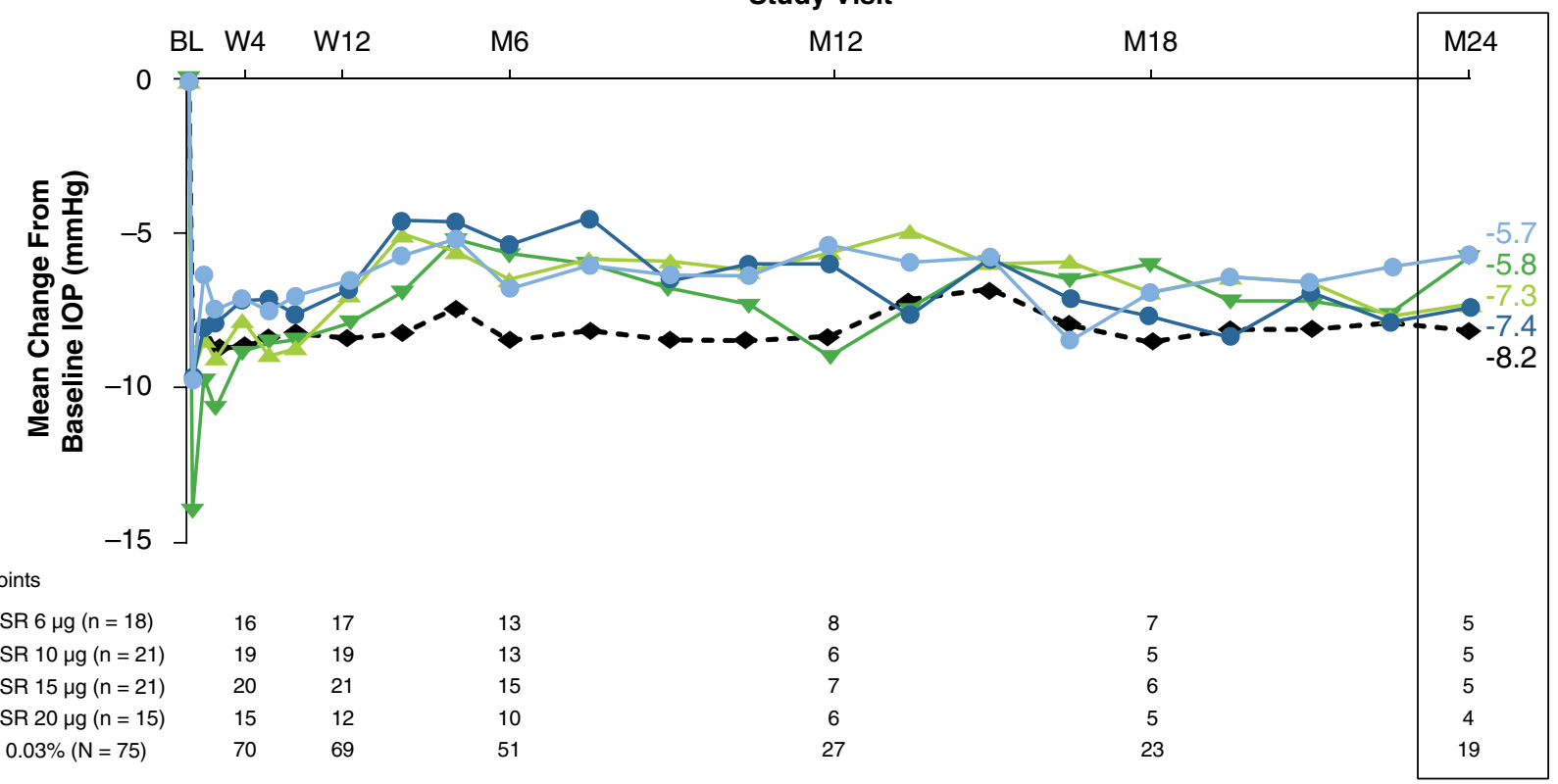

b Mean Meds=

\begin{tabular}{|c|c|c|c|c|}
\hline BimSR $6 \mu \mathrm{g}$ & 1.22 & 0 & 0.18 & 0.17 \\
\hline BimsR $10 \mu \mathrm{g}$ & 1.14 & 0 & 0 & 0 \\
\hline BimsR $15 \mu \mathrm{g}$ & 1.19 & 0 & 0 & 0 \\
\hline $\operatorname{BimSR} 20 \mu \mathrm{g}$ & 1.53 & 0 & 0 & 0.20 \\
\hline $\mathrm{mo}$ & 1.25 & 0 & 0 & 0.05 \\
\hline
\end{tabular}

$\begin{array}{ll}0.24 & 0.56 \\ 0.37 & 0.68 \\ 0.43 & 0.72 \\ 0.67 & 0.85 \\ 0.01 & 0.03\end{array}$

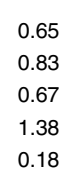

Study Visit

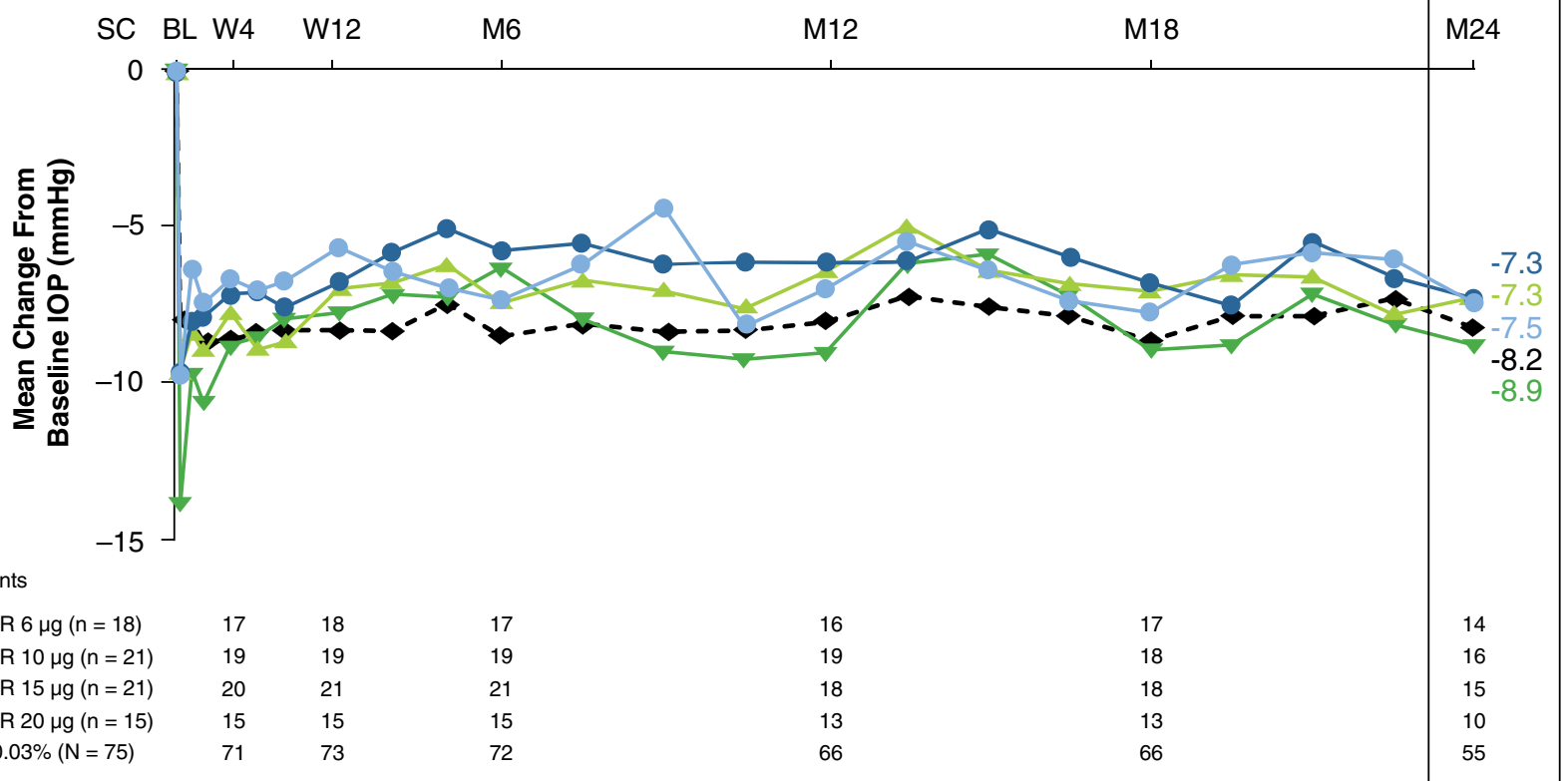

Fig. 2 Hour 0 (8:00 a.m.) mean change from baseline IOP. a Preplanned analysis with data censored after rescue or retreatment. b Analysis of all observed data. Data at month 24 were available for 55 of the 63 patients who completed the study: two patients who had not been rescued or retreated in the study eye were not evaluated within the month-24 visit window, and six patients met early exit criteria and completed the study before month 24 . By month 24, ten fellow eyes had received rescue treatment. Medication counts included every medication used except the initial implant administration in study eyes and topical bimatoprost $0.03 \%$ in fellow eyes; implant retreatment was counted as use of one medication, and fixed combinations were counted as use of two medications. Bim topical bimatoprost $0.03 \%$ once daily, BimSR bimatoprost sustained-release implant, $B L$ baseline, $I O P$ intraocular pressure, $M$ month, Meds medication counts, $S C$ screening, $W$ week 
Table 1 Average intraocular pressure reductions from baseline at study visit

\begin{tabular}{llll}
\hline Treatment & $n$ & $\begin{array}{l}\text { Overall IOP reduction } \\
\text { from baseline through } \\
\text { week 12, mmHg }\end{array}$ & $P$ value $^{\mathrm{b}}$ \\
\hline Bimatoprost SR 6 $\mu \mathrm{g}$ & 18 & $7.4 \pm 4.2$ & $<0.001$ \\
Bimatoprost SR 10 $\mu \mathrm{g}$ & 21 & $7.8 \pm 3.3$ & $<0.001$ \\
Bimatoprost SR 15 $\mu \mathrm{g}$ & 21 & $8.5 \pm 3.5$ & $<0.001$ \\
Bimatoprost SR 20 $\mu \mathrm{g}$ & 15 & $9.8 \pm 3.9$ & $<0.001$ \\
Topical bimatoprost & 75 & $8.4 \pm 3.9$ & $<0.001$ \\
0.03\% QD (pooled & & & \\
fellow eyes) & & & \\
\hline
\end{tabular}

Bimatoprost $S R$ bimatoprost sustained-release implant, IOP intraocular pressure, $Q D$ once daily

${ }^{a}$ Overall IOP reduction was calculated using all observed values for IOP reduction from baseline from day 2 through week 12, with all values weighted equally. Data are presented as mean \pm standard deviation

${ }^{\mathrm{b}} P$ value from 1 -sample $t$ test comparing the overall IOP reduction from baseline to 0

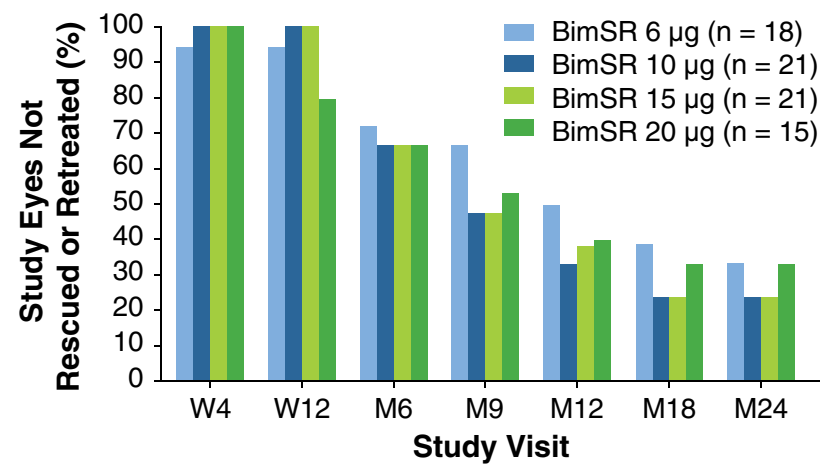

Fig. 3 Percentage of study eyes not rescued or retreated with implant. BimSR bimatoprost sustained-release implant, $M$ month, $W$ week
$75.0 \%(21 / 28)$ of the implants and $26-50 \%$ of initial size for $25.0 \%(7 / 28)$ of the implants. Analysis of residual implant at month 24 was limited to assessments for patients who did not meet criteria of total implant biodegradation allowing early study completion (at or after month 18).

Among patients who were not rescued/retreated or discontinued from the study, 28.6\% (6/21) had no visible implant in the study eye on gonioscopic examination at month 24, yet IOP remained controlled (mean IOP of $16.9 \mathrm{mmHg}$ ) at month 24 .

\subsection{Safety Outcomes}

One or more ocular AEs were reported in $64.0 \%$ of study eyes (48/75) and in $48.0 \%$ of fellow eyes (36/75) (Table 3 ). Consistent with the 6-month results [28], many of the AEs in study eyes occurred within 2 days after implant administration, i.e., within the immediate post-administration period, and were likely associated with the administration procedure itself. These AEs were typically mild and resolved quickly. The most common AE in the immediate post-administration period was conjunctival hyperemia, which could likely be attributed to the application of ophthalmic povidone-iodine during the preparation for administration.

By contrast, more than 2 days after the procedure (outside of the immediate post-administration period), AEs showed an equivalent overall incidence in the study and fellow eyes, and the incidence of some AEs typically associated with topical PGA use was lower in the eyes treated with Bimatoprost $\mathrm{SR}$ versus eyes treated with topical bimatoprost. For example, of AEs with onset later than 2 days after Bimatoprost SR administration, conjunctival hyperemia was reported in 17.3 versus $28.0 \%$; eyelash growth in 0 versus $6.7 \%$; and iris hyperpigmentation in 0 versus $4.0 \%$ of all study eyes and topically treated fellow eyes, respectively (Table 3 ).

No study eyes and two fellow eyes treated with topical bimatoprost were found to have periorbital fat atrophy upon biomicroscopic examination, and one of these findings was

Table 2 Hour 0 mean intraocular pressure $(\mathrm{mmHg}$ ) for study and fellow eyes

\begin{tabular}{llllll}
\hline Study visit & \multicolumn{2}{l}{ Bimatoprost SR } & & Bimatoprost 0.03\% \\
\cline { 2 - 5 } & $6 \mu \mathrm{g}$ & $10 \mu \mathrm{g}$ & $15 \mu \mathrm{g}$ & $20 \mu \mathrm{g}$ & $20 ; 15.2 \pm 2.0$ \\
\hline W4 & $5 ; 16.4 \pm 2.4$ & $5 ; 16.7 \pm 2.6$ & $5 ; 15.4 \pm 0.5$ & $5 ; 15.1 \pm 1.0$ & $21 ; 15.0 \pm 2.6$ \\
W12 & $6 ; 16.1 \pm 2.8$ & $5 ; 16.6 \pm 2.0$ & $5 ; 15.2 \pm 0.9$ & $5 ; 16.6 \pm 2.1$ & $21 ; 15.1 \pm 3.1$ \\
M6 & $6 ; 16.8 \pm 2.3$ & $5 ; 18.7 \pm 3.0$ & $5 ; 16.1 \pm 2.2$ & $5 ; 17.4 \pm 4.2$ & $19 ; 15.5 \pm 3.3$ \\
M12 & $5 ; 18.4 \pm 2.6$ & $4 ; 18.3 \pm 4.3$ & $5 ; 16.7 \pm 2.3$ & $5 ; 16.3 \pm 3.1$ & $21 ; 15.2 \pm 3.0$ \\
M18 & $6 ; 16.5 \pm 2.8$ & $5 ; 15.7 \pm 3.8$ & $5 ; 16.5 \pm 3.1$ & $5 ; 18.4 \pm 3.6$ & $19 ; 15.5 \pm 2.7$ \\
M24 & $5 ; 18.0 \pm 1.8$ & $5 ; 16.0 \pm 2.2$ & $5 ; 15.9 \pm 2.5$ & $4 ; 17.8 \pm 3.7$ & \\
\hline
\end{tabular}

Data are presented as $n$; mean \pm standard deviation

Study eyes without rescue or without retreatment

Bimatoprost $S R$ bimatoprost sustained-release implant, $I O P$ intraocular pressure, $W$ week, $M$ month 
Table 3 Ocular adverse events reported in two or more study eyes or fellow eyes by time of onset during the 24-month study

\begin{tabular}{|c|c|c|c|c|}
\hline \multirow[t]{2}{*}{ Adverse event } & \multicolumn{2}{|l|}{ Onset any time } & \multicolumn{2}{|c|}{ Onset $>2$ days after initial or repeat administration } \\
\hline & Bimatoprost SR $(N=75)$ & $\begin{array}{l}\text { Topical bimatoprost } \\
0.03 \%(N=75)\end{array}$ & Bimatoprost SR $(N=75)$ & $\begin{array}{l}\text { Topical bimatoprost } \\
0.03 \%(N=75)\end{array}$ \\
\hline Conjunctival hyperemia & $26(34.7)$ & $22(29.3)$ & $13(17.3)$ & $21(28.0)$ \\
\hline Foreign body sensation in eye & $13(17.3)$ & $2(2.7)$ & $2(2.7)$ & $2(2.7)$ \\
\hline Eye pain & $12(16.0)$ & 0 & $2(2.7)$ & 0 \\
\hline Conjunctival hemorrhage & $11(14.7)$ & $2(2.7)$ & $4(5.3)$ & $1(1.3)$ \\
\hline Lacrimation increased & $10(13.3)$ & 0 & $1(1.3)$ & 0 \\
\hline IOP increased & $8(10.7)$ & $2(2.7)$ & $8(10.7)$ & $2(2.7)$ \\
\hline Photophobia & $8(10.7)$ & $2(2.7)$ & $4(5.3)$ & $2(2.7)$ \\
\hline Punctate keratitis & $8(10.7)$ & $3(4.0)$ & $3(4.0)$ & $3(4.0)$ \\
\hline Vision blurred & $8(10.7)$ & $1(1.3)$ & $3(4.0)$ & $1(1.3)$ \\
\hline Dry eye & $4(5.3)$ & $4(5.3)$ & $3(4.0)$ & $4(5.3)$ \\
\hline Visual acuity reduced & $3(4.0)$ & $3(4.0)$ & $1(1.3)$ & $2(2.7)$ \\
\hline Cataract & $3(4.0)$ & $1(1.3)$ & $3(4.0)$ & $1(1.3)$ \\
\hline Conjunctivitis & $3(4.0)$ & $2(2.7)$ & $3(4.0)$ & $2(2.7)$ \\
\hline Corneal abrasion & $2(2.7)$ & 0 & 0 & 0 \\
\hline Eye irritation & $2(2.7)$ & 0 & 0 & 0 \\
\hline Eye pruritus & $2(2.7)$ & 0 & 0 & 0 \\
\hline Erythema of eyelid & $1(1.3)$ & $4(5.3)$ & $1(1.3)$ & $4(5.3)$ \\
\hline Eyelid edema & $1(1.3)$ & $2(2.7)$ & $1(1.3)$ & $2(2.7)$ \\
\hline Conjunctivitis allergic & $1(1.3)$ & $2(2.7)$ & $1(1.3)$ & $2(2.7)$ \\
\hline Growth of eyelashes & 0 & $5(6.7)$ & 0 & $5(6.7)$ \\
\hline Iris hyperpigmentation & 0 & $3(4.0)$ & 0 & $3(4.0)$ \\
\hline Eyelid retraction & 0 & $2(2.7)$ & 0 & $2(2.7)$ \\
\hline Visual field defect & 0 & $2(2.7)$ & 0 & $2(2.7)$ \\
\hline Overall $^{\mathrm{a}}$ & $48(64.0)$ & $36(48.0)$ & $35(46.7)$ & $34(45.3)$ \\
\hline
\end{tabular}

Data are presented as number of patients (\%)

Bimatoprost $S R$ bimatoprost sustained-release implant, IOP intraocular pressure

${ }^{a}$ Any ocular adverse event

reported as an AE. There were no serious ocular AEs in the study eyes, and the only serious ocular AE reported during the study was retinal detachment in a topically treated fellow eye. No treatment-related serious AEs or non-ocular AEs related to the study treatment were reported in any patient, and no patient required implant removal for safety reasons. Furthermore, no additional safety concerns were observed in patients who were retreated with a second implant or in

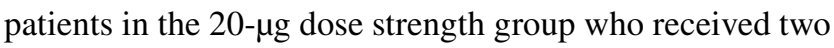
$10-\mu \mathrm{g}$ implants on day 1 .

The baseline mean \pm SD CECD was $2698.6 \pm 196.7$ and $2678.5 \pm 199.0$ cells $/ \mathrm{mm}^{2}$ in study and fellow eyes, respectively. At month 24 , the mean \pm SD CECD was $2607.0 \pm 203.0$ and $2651.6 \pm 196.4$ cells $/ \mathrm{mm}^{2}$, respectively, reflecting mean \pm SD reductions from baseline of $88.9 \pm 135.3$ and $22.2 \pm 127.8$ cells $/ \mathrm{mm}^{2}$ over 2 years in the study and fellow eyes, respectively (Fig. 4). There was no deterioration in the mean visual field mean deviation over time in Bimatoprost SR-treated eyes. At month 24, the mean $\pm \mathrm{SD}$ change in mean deviation from baseline was $+0.20 \pm 3.16 \mathrm{~dB}$ in Bimatoprost SR-treated study eyes compared with $-0.35 \pm 3.04 \mathrm{~dB}$ in topical bimatoprost-treated fellow eyes $(n=59)$ (Fig. 5). There were no notable differences between study eyes and fellow eyes in the change in central corneal thickness from baseline (Online Resource 6).

\subsection{Patient-Reported Outcomes}

Most patients reported that the procedure was somewhat or much less burdensome than expected (67-100\% across treatment groups and time points). At month 24 across all dose strengths, $82.9 \%$ of patients reported that they were extremely or very likely to have another implant procedure if given the choice, and $88.6 \%$ of patients reported they would recommend the procedure to someone else with the same eye condition (Table 4). 


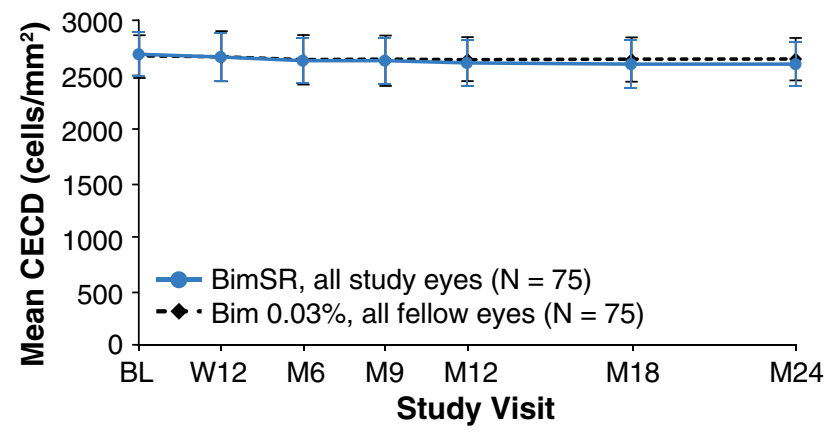

Fig. 4 Mean corneal endothelial cell density (CECD) over time in study eyes treated with one or two administrations of Bimatoprost SR $(6,10,15$, or $20 \mu \mathrm{g})$ and fellow eyes treated with daily topical bimatoprost $0.03 \%$. The between-treatment difference, i.e., study eye - fellow eye, was 20.2 (95\% CI - 12.6 to 52.9) at baseline, compared with -44.6 (95\% CI -81.6 to -7.5$)$ at month 24 . Error bars indicate the standard deviation. BimSR bimatoprost sustained-release implant, $B L$ baseline, $C I$ confidence interval, $M$ month, $W$ week

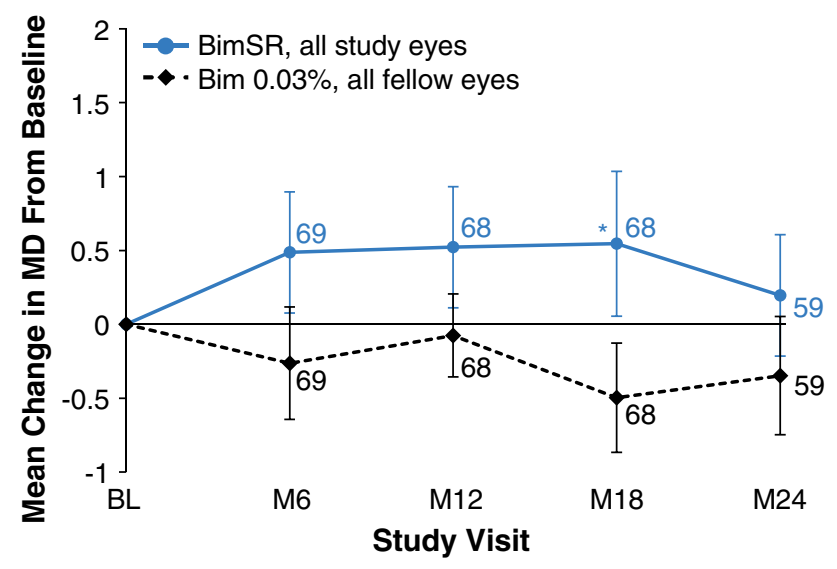

Fig. 5 Mean change in visual field mean deviation from baseline in study eyes treated with one or two administrations of Bimatoprost SR $(6,10,15$, or $20 \mu \mathrm{g})$ and fellow eyes treated with daily topical bimatoprost $0.03 \%$. Results based on observed values regardless of rescue/ retreatment; $n$ is shown in italics. Error bars indicate standard error of the mean. ${ }^{*} P=0.005$ vs. topical bimatoprost $0.03 \%$. BimSR bimatoprost sustained-release implant, $M D$ mean deviation

\section{Discussion}

This phase I/II study showed that Bimatoprost SR effectively reduced IOP for up to 24 months, had a favorable safety profile, and provided a positive treatment experience based on patient-reported outcomes. The 6-month interim analysis of this trial reported that the overall IOP-lowering efficacy of a single administration of Bimatoprost SR was similar to that of topical bimatoprost $0.03 \%$ at all assessed dose strengths $(6,10,15$, and $20 \mu \mathrm{g})$ through 16 weeks [28]. These completed trial results further show that a second administration of the implant was as effective in IOP lowering as the first administration. The efficacy of three consecutive administrations at 4-month intervals is currently being evaluated in larger populations in two phase III trials (ARTEMIS 1, NCT02247804; ARTEMIS 2, NCT02250651). As this was a multiple-dose strength efficacy and safety study, the 10and $15-\mu \mathrm{g}$ dose strengths were selected as they showed better IOP lowering than the 6- $\mu$ g dose strength in the phase I/II study and were administered as a single implant. Primary results of the phase III trials at week 12 were recently reported [29] and found to be consistent with the results of this phase I/II trial in patients followed up to 24 months.

The implant was designed to provide slow, sustained release of bimatoprost to lower IOP for 4-6 months, yet many patients experienced sustained IOP lowering for much longer than 6 months, without requiring additional treatment. A single administration of Bimatoprost SR controlled IOP in $40 \%$ of patients for up to 12 months and in $28 \%$ of patients for up to 24 months. Nonclinical pharmacokinetic studies in dogs have shown near-complete drug release and declining ocular tissue drug levels by 80 days after administration [30], with undetectable drug levels by the 4.5 -month time point (Allergan, data on file). Therefore, the duration of the IOP-lowering effect observed in many study eyes is unlikely to be due to continued drug presence.

Understanding the differences in the ocular drug distribution with Bimatoprost SR versus bimatoprost $0.03 \%$ administration may help elucidate the mechanism for the duration of the IOP-lowering effect in some patients. Nonclinical ocular pharmacokinetic studies in dogs have demonstrated a striking difference in drug distribution in eyes receiving Bimatoprost SR versus topical bimatoprost $0.03 \%$. Drug levels in off-target tissues such as eyelid margins and periorbital fat were undetectable in dog eyes treated with the implant but present in topically treated eyes [30], replicating what was previously seen with topical administration in the nonhuman primate [31]. In contrast, in target tissues such as the iris/ciliary body and the aqueous humor in dog, the maximal drug concentrations in the implant-treated eyes (51 days after administration) were approximately 4400 and 10 times higher, respectively, than in topically treated eyes (day 7) [30]. Higher drug concentrations, similar to those attained with Bimatoprost SR in the target tissues, have been shown with in vitro ciliary body cell assays to have a profound effect on the magnitude of MMP production [18]. MMP upregulation in nonhuman primates dosed with topical PGAs for 4-8 days has been demonstrated to reduce the extracellular matrix (ECM) histologically in both the ciliary muscle and the trabecular meshwork, with enlargement of the intramuscular spaces in the anterior ciliary muscle [32]. The reduction of ECM in the outflow tissues most likely reduces the hydraulic resistance to aqueous humor outflow, which lowers IOP [32-34]. 
Table 4 Patient-reported outcomes: Bimatoprost SR for glaucoma treatment

\begin{tabular}{|c|c|c|c|c|c|}
\hline & \multicolumn{5}{|c|}{ Bimatoprost SR } \\
\hline & $6 \mu \mathrm{g}(n=18)$ & $10 \mu \mathrm{g}(n=21)$ & $15 \mu \mathrm{g}(n=21)$ & $20 \mu \mathrm{g}(n=15)$ & Total $(N=75)$ \\
\hline Week 12, $n$ & 17 & 19 & 21 & 15 & 72 \\
\hline \multicolumn{6}{|c|}{ If given the choice again, are you likely to have the implant procedure? } \\
\hline Extremely likely & $10(58.8)$ & $11(57.9)$ & $12(57.1)$ & $7(46.7)$ & $40(55.6)$ \\
\hline Very likely & $4(23.5)$ & $3(15.8)$ & $6(28.6)$ & $3(20.0)$ & $16(22.2)$ \\
\hline Somewhat unlikely & $1(5.9)$ & $3(15.8)$ & 0 & $3(20.0)$ & $7(9.7)$ \\
\hline Very unlikely & 0 & $1(5.3)$ & $1(4.80)$ & $1(6.7)$ & $3(4.2)$ \\
\hline Extremely unlikely & $2(11.8)$ & $1(5.3)$ & $2(9.5)$ & $1(6.7)$ & $6(8.3)$ \\
\hline \multicolumn{6}{|c|}{ Are you likely to recommend the implant to someone else with your eye condition? } \\
\hline Extremely likely & $11(64.7)$ & $12(63.2)$ & $13(61.9)$ & $7(46.7)$ & $43(59.7)$ \\
\hline Very likely & $4(23.5)$ & $3(15.8)$ & $6(28.6)$ & $4(26.7)$ & $17(23.6)$ \\
\hline Somewhat unlikely & $1(5.9)$ & $2(10.5)$ & 0 & $3(20.0)$ & $6(8.3)$ \\
\hline Very unlikely & 0 & 0 & $1(4.8)$ & $1(6.7)$ & $2(2.8)$ \\
\hline Extremely unlikely & $1(5.9)$ & $2(10.5)$ & $1(4.8)$ & 0 & $4(5.6)$ \\
\hline Month 24, $n$ & 16 & 20 & 20 & 14 & 70 \\
\hline \multicolumn{6}{|c|}{ If given the choice again, are you likely to have the implant procedure? } \\
\hline Extremely likely & $9(56.3)$ & $8(40.0)$ & $11(55.0)$ & $7(50.0)$ & $35(50.0)$ \\
\hline Very likely & $5(31.3)$ & $8(40.0)$ & $7(35.0)$ & $3(21.4)$ & $23(32.9)$ \\
\hline Somewhat unlikely & $2(12.5)$ & $4(20.0)$ & 0 & $3(21.4)$ & $9(12.9)$ \\
\hline Very unlikely & 0 & 0 & $1(5.0)$ & 0 & $1(1.4)$ \\
\hline Extremely unlikely & 0 & 0 & $1(5.0)$ & $1(7.1)$ & $2(2.9)$ \\
\hline \multicolumn{6}{|c|}{ Are you likely to recommend the implant to someone else with your eye condition? } \\
\hline Extremely likely & $9(56.3)$ & $10(50.0)$ & $14(70.0)$ & $7(50.0)$ & $40(57.1)$ \\
\hline Very likely & $6(37.5)$ & $6(30.0)$ & $4(20.0)$ & $6(42.9)$ & $22(31.4)$ \\
\hline Somewhat unlikely & $1(6.3)$ & $4(20.0)$ & $1(5.0)$ & 0 & $6(8.6)$ \\
\hline Very unlikely & 0 & 0 & 0 & 0 & 0 \\
\hline Extremely unlikely & 0 & 0 & $1(5.0)$ & $1(7.1)$ & $2(2.9)$ \\
\hline
\end{tabular}

Data are presented as $N(\%)$

Bimatoprost $S R$ bimatoprost sustained-release implant

Another study in the same nonhuman primate model treated topically for 1 year with bimatoprost $0.03 \%$ showed similar morphologic changes, with channel-like structures forming in the anterior ciliary muscle (with partial endothelial cell lining of the channels) after chronic dosing [14]. In the trabecular meshwork, remodeling included disruption of the endothelial cell monolayer of Schlemm's canal, expansion of the juxtacanalicular region of the trabecular meshwork, and loss of ECM, as well as widening of intertrabecular spaces in the corneoscleral region of the trabecular meshwork in some samples [14]. Given this information, we postulate that the duration of the IOP-lowering effect with Bimatoprost SR could potentially be explained by the higher target tissue (iris-ciliary body, aqueous humor) drug concentrations following administration [30], resulting in higher MMP upregulation and more durable tissue remodeling.

The long-term safety/tolerability profile of one or two implants over 2 years of follow-up was consistent with the 6-month interim results [28] and remain favorable in this study. AEs that occurred more frequently in Bimatoprost SRtreated study eyes than in topical bimatoprost $0.03 \%$-treated fellow eyes (e.g., conjunctival hyperemia, foreign body sensation, eye pain) typically occurred within the immediate post-administration period (within 2 days after administration) and were likely procedure related (e.g., related to the sterile administration procedure preparation). Beyond the immediate post-administration period, the overall incidence of AEs was similar in the Bimatoprost SR-treated eyes and the topically treated fellow eyes, with some AEs typically associated with PGA use (such as eyelash growth) reported more frequently in the topically treated fellow eyes than in the Bimatoprost SR-treated eyes. The reduced incidence in the implant-treated eyes of AEs typically associated with topical PGA use suggests that Bimatoprost SR targets drug delivery to its relevant sites of action, reducing exposure of bimatoprost to the ocular surface and surrounding off-target 
tissues [30]. Furthermore, the CECD results were similar for the study eyes and the fellow eyes, with no clinically significant mean changes in CECD seen during the study. Most patients reported a favorable treatment experience with Bimatoprost SR and were extremely or very likely to have the implant procedure and to recommend it.

Glaucoma is a chronic, slowly progressing disease with a high and increasing global prevalence [3]; the risk of blindness increases if IOP is not controlled. In some patients, IOPs are not controlled with conventional topical therapy mainly because of nonadherence. Thus, there is a large unmet need for new, effective, drop-free, and well-tolerated IOP-lowering drug-delivery options that can reduce the patient's self-administered treatment burden and present a favorable patient experience while helping minimize the risk of vision loss from glaucoma [22]. By providing sustained IOP-lowering effects, drug-delivery systems such as Bimatoprost SR may help to address this unmet need. These new delivery systems may delay the need for more invasive (surgical) treatment by more precisely targeting the drug to sites of action [30, 35, 36], while providing IOP-lowering effects and tolerability profiles comparable to those of topical therapy $[28,37,38]$. Other sustained-release products are in various phases of clinical development, including ENV515 (Envisia Therapeutics, Durham, NC, USA), OTXTIC (Ocular Therapeutix, Bedford, MA, USA), and iDose (Glaukos Corporation, Laguna Hills, CA, USA) for intracameral delivery of travoprost. The BIM Ring (Allergan plc, Dublin, Ireland) is being developed for sustained delivery of bimatoprost to the ocular surface.

Limitations of the current study may include the selection of patients who had previously responded to a topical PGA with at least $20 \%$ IOP lowering; the results may not be generalizable to patients who have not been previously treated with a PGA. It should also be noted that bimatoprost $0.03 \%$ ophthalmic solution was used as the active control, as it was the formulation in use at the time of initiation of the study, and the efficacy observed in the topical treatment arm was consistent with the 28-33\% IOP lowering typically reported in studies of topical bimatoprost $0.03 \%$ [39]. The most widely used topical ophthalmic formulation of bimatoprost currently available for use in the USA is bimatoprost $0.01 \%$ ophthalmic solution, which demonstrates similar efficacy to and improved tolerability compared with the original bimatoprost $0.03 \%$ formulation [19]. Another potential study limitation is that $71 \%$ of patients enrolled were White; whether the IOP-lowering effectiveness of Bimatoprost SR varies with race is unknown. However, the population distribution by study location suggests that this representation reflects overall population demographics, particularly in the USA [40].

Bimatoprost SR demonstrated favorable efficacy and safety after one or two administrations over 2 years of follow-up. Results obtained in this phase I/II study suggest that Bimatoprost SR may represent a transformational new approach to IOP-lowering therapy, with some patients exhibiting sustained IOP-lowering effects up to 2 years after a single administration. Bimatoprost SR has the potential to reduce patient treatment burden and ensure adherence with minimal adverse effects. The present results support further clinical development of Bimatoprost SR.

Acknowledgements Writing and editorial assistance was provided to the authors by Scarlett Geunes-Boyer, PhD, of Evidence Scientific Solutions, Inc (Philadelphia, PA, USA) and Tina Treece, PhD (Allergan plc), and funded by Allergan plc, Dublin, Ireland. All authors met the ICMJE authorship criteria. Neither honoraria nor payments were made for authorship.

Data Availability Allergan will share de-identified patientlevel data and study-level data, including protocols and clinical study reports, for phase II, III, or IV trials completed after 2008 that are registered to ClinicalTrials.gov or EudraCT, have received regulatory approval in the USA and/ or the EU in a given indication and the primary manuscript from the trial has been published. To request access to the data, the researcher must sign a data use agreement, and any shared data are to be used for non-commercial purposes. More information can be found on http://www.allergancl inicaltrials.com/.

Bimatoprost SR Study Group Tin Aung, FRCSEd, PhD (Singapore); Allen D. Beck, MD (Atlanta, GA, USA); James D. Branch, MD (Winston-Salem, NC, USA); William C. Christie, MD (Cranberry Township, PA, USA); Michael Coote, MBBS, FRANZCO (Fitzroy, VIC, Australia); Charles J. Crane, MD (South Orange, NJ, USA); E. Randy Craven, MD (Parker, CO, USA); Andrew Crichton, MD (Calgary, AB, Canada); Douglas G. Day, MD (Roswell, GA, USA); Steven Day, MD (Spokane, WA, USA); F. Jane Durcan, MD (Spokane, WA, USA); Richard M. Evans, MD (San Antonio, TX, USA); William J. Flynn, MD (San Antonio, TX, USA); Sébastien Gagné, MD, FRCSC (Boisbriand, QC, Canada); Damien F. Goldberg, MD (Torrance, CA, USA); Jack V. Greiner, OD, DO, PhD (Winchester, MA, USA); Paul Jeppsen, MD (Salt Lake City, UT, USA); Delan Jinapriya, MD (Kingston, ON, Canada); C. Starck Johnson, MD (Parker, CO, USA); Shimon Kurtz, MD (Tel Aviv, Israel); Richard A. Lewis, MD (Sacramento, CA, USA); Steven L. Mansberger, MD, MPH (Portland, OR, USA); Joseph R. Martel, MD (Rancho Cordova, CA, USA); Shamira A. Perera, MBBS, FRCOphth (Singapore); Michael H. Rotberg, MD (Charlotte, NC, USA); Robert M. Saltzmann, MD (Charlotte, NC, USA); Howard I. Schenker, MD (Rochester, NY, USA); Michael E. Tepedino, MD (High Point, NC, 
USA); Maria Imelda R. Yap-Veloso, MD (Makati City, Philippines); Harvey S. Uy, MD (Makati City, Philippines); and Thomas R. Walters, MD (Austin, TX, USA).

\section{Compliance with Ethical Standards}

Ethics approval Institutional review board or independent ethics committee approval was obtained at each site before the study began, and the study was performed in compliance with good clinical practice, the principles of the Declaration of Helsinki, or the laws and regulations of the country in which the study was conducted (ClinicalTrials.gov registration number NCT01157364).

Informed consent All patients in this study provided written informed consent before undergoing any study-related procedure.

Funding This study was sponsored by Allergan plc (Dublin, Ireland). The study sponsor participated in the design of the study, data management, analysis and interpretation, and preparation, review, and approval of the manuscript.

Conflict of interest E. Randy Craven is a consultant for Aerie, Alcon, Allergan, Ivantis, Santen, and WL Gore and has received grant support from Allergan. Thomas Walters is a consultant for Allergan, Nicox, Ocular Therapeutix, and Sun. William C. Christie is a consultant for Allergan and Johnson \& Johnson. Douglas G. Day is a consultant for Allergan. Richard A. Lewis is a consultant for Aerie, Alcon, Allergan, Aquesys, AVS, Envisia, Glaukos, Ivantis, Oculeve, PolyActiva, ViSci, and Zeiss. Margot L. Goodkin, Michelle Chen, Veronica Wangsadipura, Michael R. Robinson, and Marina Bejanian are Allergan employees.

Open Access This article is licensed under a Creative Commons Attribution-NonCommercial 4.0 International License, which permits any non-commercial use, sharing, adaptation, distribution and reproduction in any medium or format, as long as you give appropriate credit to the original author(s) and the source, provide a link to the Creative Commons licence, and indicate if changes were made. The images or other third party material in this article are included in the article's Creative Commons licence, unless indicated otherwise in a credit line to the material. If material is not included in the article's Creative Commons licence and your intended use is not permitted by statutory regulation or exceeds the permitted use, you will need to obtain permission directly from the copyright holder. To view a copy of this licence, visit http://creativecommons.org/licenses/by-nc/4.0/.

\section{References}

1. American Academy of Ophthalmology. Preferred practice pattern in primary open-angle glaucoma; 2016. https://www.aao.org/prefe rred-practice-pattern/primary-open-angle-glaucoma-ppp-2015. Accessed Sep 17, 2019.

2. European Glaucoma Society. Terminology and guidelines for glaucoma (4th edition); 2014. http://www.eugs.org/eng/EGS_ guidelines4.asp. Accessed Oct 18, 2016.

3. Quigley HA, Broman AT. The number of people with glaucoma worldwide in 2010 and 2020. Br J Ophthalmol. 2006;90:262-7.

4. Tham YC, Li X, Wong TY, Quigley HA, Aung T, Cheng CY. Global prevalence of glaucoma and projections of glaucoma burden through 2040: a systematic review and meta-analysis. Ophthalmology. 2014;121:2081-90.

5. McKean-Cowdin R, Varma R, Wu J, Hays RD, Azen SP. Severity of visual field loss and health-related quality of life. Am J Ophthalmol. 2007;143:1013-23.

6. Thygesen J, Aagren M, Arnavielle S, Bron A, Frohlich SJ, Baggesen K, et al. Late-stage, primary open-angle glaucoma in Europe: social and health care maintenance costs and quality of life of patients from 4 countries. Curr Med Res Opin. 2008;24:1763-70.

7. Varma R, Lee PP, Goldberg I, Kotak S. An assessment of the health and economic burdens of glaucoma. Am J Ophthalmol. 2011;152:515-22.

8. Ramulu PY, Mihailovic A, West SK, Gitlin LN, Friedman DS. Predictors of falls per step and falls per year at and away from home in glaucoma. Am J Ophthalmol. 2019;200:169-78.

9. Miller AB, Lajoie K, Strath RA, Neima DR, Marigold DS. Coordination of gaze behavior and foot placement during walking in persons with glaucoma. J Glaucoma. 2018;27:55-63.

10. Wang Y, Alnwisi S, Ke M. The impact of mild, moderate, and severe visual field loss in glaucoma on patients' quality of life measured via the Glaucoma Quality of Life-15 Questionnaire: a meta-analysis. Medicine (Baltimore). 2017;96:e8019.

11. Tamm ER. The trabecular meshwork outflow pathways: structural and functional aspects. Exp Eye Res. 2009;88:648-55.

12. Lim KS, Nau CB, O’Byrne MM, Hodge DO, Toris CB, McLaren $\mathrm{JW}$, et al. Mechanism of action of bimatoprost, latanoprost, and travoprost in healthy subjects. A crossover study. Ophthalmology. 2008;115(790-5):e4.

13. Kim JW, Lindsey JD, Wang N, Weinreb RN. Increased human scleral permeability with prostaglandin exposure. Invest Ophthalmol Vis Sci. 2001;42:1514-21.

14. Richter M, Krauss AH, Woodward DF, Lutjen-Drecoll E. Morphological changes in the anterior eye segment after long-term treatment with different receptor selective prostaglandin agonists and a prostamide. Invest Ophthalmol Vis Sci. 2003;44:4419-26.

15. Winkler NS, Fautsch MP. Effects of prostaglandin analogues on aqueous humor outflow pathways. J Ocul Pharmacol Ther. 2014;30:102-9.

16. Woodward DF, Gil DW. The inflow and outflow of anti-glaucoma drugs. Trends Pharmacol Sci. 2004;25:238-41.

17. Oh DJ, Martin JL, Williams AJ, Russell P, Birk DE, Rhee DJ. Effect of latanoprost on the expression of matrix metalloproteinases and their tissue inhibitors in human trabecular meshwork cells. Invest Ophthalmol Vis Sci. 2006;47:3887-95.

18. Yamada H, Yoneda M, Gosho M, Kato T, Zako M. Bimatoprost, latanoprost, and tafluprost induce differential expression of matrix metalloproteinases and tissue inhibitor of metalloproteinases. BMC Ophthalmol. 2016;16:26.

19. Katz LJ, Cohen JS, Batoosingh AL, Felix C, Shu V, Schiffman RM. Twelve-month, randomized, controlled trial of bimatoprost $0.01 \%, 0.0125 \%$, and $0.03 \%$ in patients with glaucoma or ocular hypertension. Am J Ophthalmol. 2010;149:661-71.

20. Robin AL, Novack GD, Covert DW, Crockett RS, Marcic TS. Adherence in glaucoma: objective measurements of oncedaily and adjunctive medication use. Am J Ophthalmol. 2007; 144:533-40.

21. Yeaw J, Benner JS, Walt JG, Sian S, Smith DB. Comparing adherence and persistence across 6 chronic medication classes. J Manag Care Pharm. 2009;15:728-40.

22. Sleath B, Blalock S, Covert D, Stone JL, Skinner AC, Muir K, et al. The relationship between glaucoma medication adherence, eye drop technique, and visual field defect severity. Ophthalmology. 2011;118:2398-402.

23. Reardon G, Kotak S, Schwartz GF. Objective assessment of compliance and persistence among patients treated for glaucoma and 
ocular hypertension: a systematic review. Patient Prefer Adherence. 2011;5:441-63.

24. Schwartz GF, Hollander DA, Williams JM. Evaluation of eye drop administration technique in patients with glaucoma or ocular hypertension. Curr Med Res Opin. 2013;29:1515-22.

25. Stryker JE, Beck AD, Primo SA, Echt KV, Bundy L, Pretorius $\mathrm{GC}$, et al. An exploratory study of factors influencing glaucoma treatment adherence. J Glaucoma. 2010;19:66-72.

26. Newman-Casey PA, Robin AL, Blachley T, Farris K, Heisler $\mathrm{M}$, Resnicow K, et al. The most common barriers to glaucoma medication adherence: a cross-sectional survey. Ophthalmology. 2015;122:1308-16.

27. Lee SS, Hughes P, Ross AD, Robinson MR. Biodegradable implants for sustained drug release in the eye. Pharm Res. 2010;27:2043-53.

28. Lewis RA, Christie WC, Day DG, Craven ER, Walters T, Bejanian $\mathrm{M}$, et al. Bimatoprost sustained-release implants for glaucoma therapy: 6-month results from a phase I/II clinical trial. Am J Ophthalmol. 2017;175:137-47.

29. Craven ER, Walters T, Christie W, Bejanian M, Goodkin ML, Guo Q, Zhang J, Robinson MR, Ahmed IK. Phase 3 evaluation of Bimatoprost sustained-release implant in patients with glaucoma or ocular hypertension: results at primary database lock [abstract no. PA054-2019]. Presented at the American Academy of Ophthalmology 2019 meeting, San Francisco, CA, 12-15 October 2019. https://aao.scientificposters.com/epsAbstractAAO. cfm?id=2. Accessed 23 Dec 2019.

30. Seal JR, Robinson MR, Burke J, Bejanian M, Coote M, Attar M. Intracameral sustained-release bimatoprost implant delivers bimatoprost to target tissues with reduced drug exposure to offtarget tissues. J Ocul Pharmacol Ther. 2019;35:50-7.

31. Woodward DF, Krauss AH, Chen J, Liang Y, Li C, Protzman $\mathrm{CE}$, et al. Pharmacological characterization of a novel antiglaucoma agent, Bimatoprost (AGN 192024). J Pharmacol Exp Ther. 2003;305:772-85.
32. Lutjen-Drecoll E, Tamm E. Morphological study of the anterior segment of cynomolgus monkey eyes following treatment with prostaglandin F2 alpha. Exp Eye Res. 1988;47:761-9.

33. De Groef L, Andries L, Siwakoti A, Geeraerts E, Bollaerts I, Noterdaeme L, et al. Aberrant collagen composition of the trabecular meshwork results in reduced aqueous humor drainage and elevated IOP in MMP-9 null mice. Invest Ophthalmol Vis Sci. 2016;57:5984-95.

34. Bradley JM, Vranka J, Colvis CM, Conger DM, Alexander JP, Fisk AS, et al. Effect of matrix metalloproteinases activity on outflow in perfused human organ culture. Invest Ophthalmol Vis Sci. 1998;39:2649-58.

35. Kim J, Kudisch M, da Silva NRK, Asada H, Aya-Shibuya E, Bloomer MM, et al. Long-term intraocular pressure reduction with intracameral polycaprolactone glaucoma devices that deliver a novel anti-glaucoma agent. J Control Release. 2018;269:45-51.

36. Kim J, Kudisch M, Mudumba S, Asada H, Aya-Shibuya E, Bhisitkul RB, et al. Biocompatibility and pharmacokinetic analysis of an intracameral polycaprolactone drug delivery implant for glaucoma. Invest Ophthalmol Vis Sci. 2016;57:4341-6.

37. Aref AA. Sustained drug delivery for glaucoma: current data and future trends. Curr Opin Ophthalmol. 2017;28:169-74.

38. Brandt JD, DuBiner HB, Benza R, Sall KN, Walker GA, Semba CP. Long-term safety and efficacy of a sustained-release bimatoprost ocular ring. Ophthalmology. 2017;124:1565-6.

39. van der Valk R, Webers CA, Schouten JS, Zeegers MP, Hendrikse F, Prins MH. Intraocular pressure-lowering effects of all commonly used glaucoma drugs: a meta-analysis of randomized clinical trials. Ophthalmology. 2005;112:1177-85.

40. United States Census Bureau. United States Census 2018: QuickFacts-United States; 2018. https://www.census.gov/quickfacts/ fact/table/US/PST045218. Accessed Nov 1, 2019. 